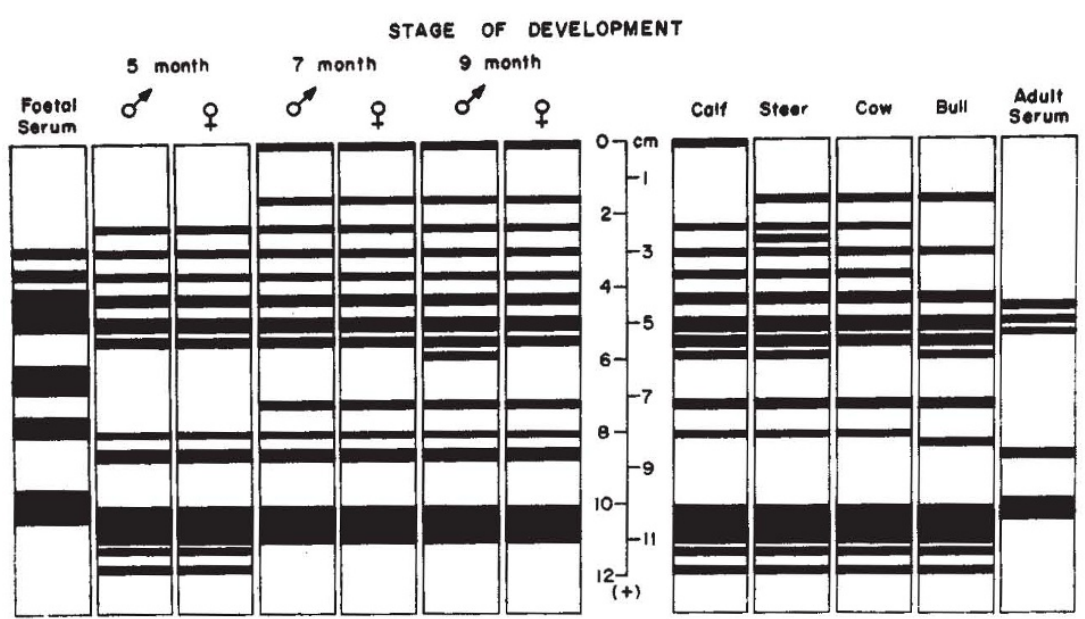

Fig. 1. Electrophoretic patterns of aorta glycoprotein. A representation of glycoprotein components isolated from aortas at the stages indicated. Tissues from at least twelve aortas were pooled to obtain the results, with the exception of the 9 month foetuses where eight aortas of each sex were pooled and of the calves where six were used. The patterns to th left of the scale are foetal. Serum patterns are included for comparison. The anodic end is

similarity between these patterns and those of the aorta glycoprotein fractions.

A sex difference in glycoprotein composition of cattle aorta comparable with that of humans ${ }^{6}$ has been observed. Pooling of the aortas into groups minimizes individual variations, and accentuates the changes which seem to be functions of development and ageing. It is difficult to interpret these results without considering complicated sex linked genetic ${ }^{9}$ and physiological factors ${ }^{10,11}$. It is clear, however, that investigators concerned with the isolation of macromolecular components of connective tissue should devote more attention to the age and sex of the source.

The work was supported by grants from NIH and NASA. We thank Peyton Packing Co., Inc., Texas, for providing the samples.

Department of Chemistry,

New Mexico State University,

Las Cruces. New Mexico.

Received October 23, 1967; revised March 11, 1968.

1 Buddeke, E., Z. Physiol. Chem., 318, 33 (1960).

2 Berenson, G. S., and Fishkin, A. F., Arch. Biochem. Biophys., 97, 18 (1962).

- Radhakrishnamurthy, B., Fishkin, A. F., Hubbell, G. J., and Berenson, G. S., Arch. Biochem. Biophys., 104, 19'(1964).

- Radhakrishnamurthy, B., Fishkin, A. F., and Berenson, G. S., Biochim. Biophys. Acta, 101, 129 '(1965).

- Radhakrishnamurthy, B., and Berenson, G. S., J. Biol. Chem., 241, 2106 (1966).

- Berenson, G. S., Radhakrishnamurthy, B., Fishkin, A. F., Dessauer, H., and Arquembourg, P., J. Atheroscler. Res., 6, 214 (1966).

'Arthur, G. H., Wright's Veterinary Obstetrics, third ed., 44 (The Williams and Wilkins Co., Baltimore, 1964).

- Fishkin, A. F., and Berenson, G. S., Arch. Biochem. Biophys., 95, 130 (1961).

- Stern, C., Amer. J. Hum. Genet., 9, 147 (1957).

10 Asboe-Hansen, G., Fed. Proc., 25, 1136 (1966).

11 Catchpole, H. R., Fed. Proc., 25, 1144 (1966).

\section{Biosynthesis of Complex Terpenes in the Leaf Cuticle and Trichomes of Nicotiana tabacum}

THE cuticle of the leaves of Nicotiana tabacum is covered with a gummy layer, some of which seems to originate as an exudate from the leaf hairs or trichomes.

Various observations suggest that precursors of the characteristic aroma of tobacco may be derived from the leaf trichomes. Varieties known for their high quality and aroma are "gummier" than low quality varieties, and clothing smeared with leaf exudate while working in the field develops the aroma of cured tobacco on storage ${ }^{1}$.
The inflorescence of Nicotiana has a dense population of trichomes and is covered with exudate. A concentrate of complex terpenes can be readily obtained, by washing the inflorescence with benzene.

The polar lipids can be removed from the outside of the leaf by washing in solvent and consist of a complex mixture of non-volatile terpenes, which can be separated by thin-layer chromatography ('Kieselgel $G$ ' and 5 per cent methanol in chloroform) and gasliquid chromatography of the trimethyl silyl ethers. The components are chiefly terpene diols in which the $\alpha$ and $\beta$ isomers of 12-isopropyl-1,5,9trimethyl - 4,8,13 - cyclotetradecatriene-1,3-diol (ref. 2) predominate.

Cuticle was peeled from the underside of young leaves of $N$. tabacum under a dissecting microscope, and trichomes were obtained by carefully shaving the stems of mature plants. Both cuticles bearing trichomes and trichomes alone were suspended in small volumes of buffer containing ${ }^{14} \mathrm{C}$-2-acetate or ${ }^{14} \mathrm{C}$-2-mevalonate and maintained for periods of 15-90 h in an illuminated growth chamber.

In every case, labelled terpenoids were produced. Preliminary studies have shown that the incorporated radioactivity moves from one component to another in an as yet undefined biosynthetic pattern.

The biosynthesis can be inhibited by tris-(2-diethylaminoethyl)-phosphate hydrochloride. This compound also inhibits phytosterol synthesis in $N$. tabacum ${ }^{3}$ with accumulation of squalene 2,3-oxide (ref. 4). Biosynthetically prepared ${ }^{14} \mathrm{C}$-squalene 2,3-oxide (unpublished work of W. W. R.) applied to the surface of $N$. tabacum leaves is chiefly incorporated into 4,4-dimethyl, $4 \alpha$-methyl and 4-demethyl sterols, but part of the radioactivity is found in some compounds of the polar terpenoid group.

The terpenoid biosynthesis found in detached trichomes is of particular interest because these organelles have a terminal cell containing chloroplasts which stains intensely with reagents reacting with terpenes.

\section{MaRgareT J. Michie} W. W. REID*

Research Department,

British Tobacco Co. (Australia) Ltd,

Sydney, NSW, Australia.

Received January 12; revised March 15, 1968.

* Present address: Department of Organic Chemistry, University of Western Australia, Nedlands, WA.

1 Barrera, R., and Wernsman, E. A., Tobacco Sci., 10, 157 (1966).

${ }^{2}$ Roberts, P. L., and Rowland, R. L., J. Org. Chem., 27, 3984 (1962).

${ }^{3}$ Reid, W. W., Biochem. J., 100, $13 P$ (1966).

${ }^{4}$ Reid, W. W., Phytochemistry, 7, 451 (1968).

\section{Characterization of Natural Inhibitors of Trypsin and Chymotrypsin by Electrophoresis in Acrylamide- Agarose Gels}

We have detected natural inhibitors of enzymes in microgram quantities. In our method, after gel electrophoresis of a sample presumed to contain inhibitory activity, the slab of gel was incubated in a solution of the appropriate enzyme which then entered the gel by diffusion and formed a thin and homogeneous layer on its surface. After several minutes the gel was removed from the solution, allowed to stand until completion of the enzyme-inhibitor complex, and then transferred into a solution containing a chromogenic substrate for the enzyme used in the assay. 